\title{
HAUSDORFF MEASURE OF THE SINGULAR SET OF QUASIREGULAR MAPS ON CARNOT GROUPS
}

\author{
IRINA MARKINA
}

Received 14 December 2002

\begin{abstract}
Recently, the theory of quasiregular mappings on Carnot groups has been developed intensively. Let $v$ stand for the homogeneous dimension of a Carnot group and let $m$ be the index of the last vector space of the corresponding Lie algebra. We prove that the $(v-m-1)$-dimensional Hausdorff measure of the image of the branch set of a quasiregular mapping on the Carnot group is positive. Some estimates of the local index of quasiregular mappings are also obtained.
\end{abstract}

2000 Mathematics Subject Classification: 22E30, 30C65, 43A80.

1. Introduction and statement of main results. Quasiregular mappings, or, in another terminology, mappings with bounded distortion, were firstly introduced and investigated by Reshetnyak in a series of papers that began to appear in 1966 [29, 31]. His work was handed and furthered by several analysts, among them Martio, Rickman, Väisälä [22, 23, 32], and others [1, 28, 33] later. The analytic definition of a quasiregular mapping is similar to the analytic definition of a quasiconformal one, with the exception of the requirement of homeomorphism.

Recently, the analysis on homogeneous groups (the Carnot groups) has been developed intensively. The fundamental role of such groups in analysis was pointed out by Stein [34] in his address to the International Congress of Mathematicians in 1970 (see also his monograph [35]). Briefly, a homogeneous group is a simply connected nilpotent Lie group, whose Lie algebra admits a grading. There is a natural family of dilations on the group under which the metric behaves like the Euclidean metric under the Euclidean dilation [7, 10]. The analysis on the homogeneous group is also a test ground for the study of general subelliptic problems arising from vector fields $X_{1}, \ldots, X_{k}$ satisfying the Hörmander hypoellipticity condition [17].

Quasiconformal mappings on a homogeneous group of a special type were initially considered by Mostow [24] in 1971 in connection with rigidity theorems for the rank one symmetric space. Various definitions of quasiconformal mappings can be found in $[12,15,18,37,38,39]$. In the works $[13,16,19,20$, $37,39,40,42]$, analytical foundations of the theory of quasiregular mappings 
on Carnot groups were developed. We should note that in $[13,16]$ quasiregular mappings were studied with an additional smoothness condition. An alternative approach to this theory on the Heisenberg group was given in the works $[8,9]$.

The next analytic definition of a quasiregular mapping on a Carnot group $\mathbb{G}$ is the basic in this paper.

Definition 1.1. Let $\Omega \subset \mathbb{G}$ be a domain. A mapping $f: \Omega \rightarrow \mathbb{G}$ is said to be a quasiregular mapping (mapping with bounded distortion) if

(1) $f$ is continuous open and discrete,

(2) $f$ belongs to $H W_{v, \text { loc }}^{1}(\Omega)$,

(3) the formal horizontal differential $D_{H} f$ satisfies the condition

$$
\left|D_{H} f(x)\right|^{v} \leq K J(x, f)
$$

for almost all $x \in \Omega$.

The smallest constant $K$ is called the outer coefficient of quasiregularity and is denoted by $K_{O}(f)$. If $f: \Omega \rightarrow \mathbb{G}$ is a quasiregular mapping, then there is a constant $K^{\prime}$ such that

$$
J(x, f) \leq K^{\prime}\left(\inf _{\xi \in V_{1}, \rho(\xi)=1} \rho\left(D_{H} f(x)(\xi)\right)\right)^{v}
$$

for almost all $x \in \Omega$. The smallest constant $K^{\prime}$ is called the inner coefficient of quasiregularity and is denoted by $K_{I}(f)$. The relationships $K_{O}(f) \leq C K_{I}^{v-1}(f)$ and $K_{I}(f) \leq C K_{O}^{\nu-1}(f)$ are realized, where $C$ is some constant. In the case $\mathbb{G}=$ $\mathbb{R}^{n}$, the constant $C$ is equal to 1 . Also we use the notation $K(f)=\max \left\{K_{O}(f)\right.$, $\left.K_{I}(f)\right\}$ and call $K(f)$ the coefficient of quasiregularity. If $\mathbb{G}=\mathbb{R}^{n}$, then Definition 1.1 is compatible with the definition of the quasiregular mapping in the Euclidean space. The definition of $D_{H} f$ and more details can be found in Section 2.

We denote by $B_{f}$ the branch set of $f$, that is,

$$
B_{f}=\{x \mid f \text { is not locally homeomorphic at } x\} .
$$

A result by Černavskiǐ [2, 3] says that a discrete open map $f$ in $\mathbb{R}^{n}$ satisfies $\operatorname{dim} B_{f}=\operatorname{dim} f\left(B_{f}\right) \leq n-2$. Homotopy considerations (see [23]) imply that the $(n-2)$-dimensional Hausdorff measure of $f\left(B_{f}\right)$ is strictly positive. By definition, a quasiregular mapping on the Carnot group is a discrete open map, that yields immediately a similar inequality for the set $f\left(B_{f}\right)$. In the present paper, we give a more precise estimate for the Hausdorff measure of $f\left(B_{f}\right)$, namely, we prove the next theorem.

THEOREM 1.2. Let $\Omega \subset \mathbb{G}$ be an open connected set, and let $f: \Omega \rightarrow \mathbb{G}$ be a nonconstant quasiregular mapping such that the set of branch points $B_{f}$ is not 
empty. Then

$$
\mathscr{H}_{\rho}^{\nu-(m+1)}\left(f\left(B_{f}\right)\right)>0 .
$$

Here $m$ is an index of the last vector space in the graduated Lie algebra 9 of $\mathbb{G}$.

Some estimates for the local index of quasiregular mappings on Carnot groups are also obtained.

2. Notes and preliminary results. Let $\mathscr{G}$ be a Lie algebra and let $\mathbb{G}$ be a corresponding simply connected Lie group. If $U$ and $V$ are some sets from $\mathscr{G}$, then we denote by $[U, V]$ the subspace of the algebra $\mathscr{G}$ generated by the elements $[X, Y]=X Y-Y X, X \in U, Y \in V$. By induction, we define the following series:

$$
\mathscr{G}_{1}=\mathscr{G}, \quad \mathscr{G}_{j}=\left[\mathscr{G}_{,} \mathscr{G}_{j-1}\right] ; \quad \mathbb{G}_{1}=\mathbb{G}, \quad \mathbb{G}_{j}=\left[\mathbb{G}, \mathbb{G}_{j-1}\right] .
$$

A Lie algebra is called nilpotent of step $m$ if $\mathscr{G}_{m+1}=\{0\}$, but $\mathscr{G}_{m} \neq\{0\}$.

We call a Lie algebra to be graduated if it splits into the direct sum of vector spaces $\varphi=V_{1} \oplus V_{2} \oplus \cdots \oplus V_{k} \oplus \cdots$. Here $\left[V_{i}, V_{j}\right] \subset V_{i+j}$, and only finitely many elements do not vanish. A Lie algebra $\mathscr{G}$ is called stratified if $\mathscr{G}$ is graduated and the subspace $V_{1} \subset \mathscr{G}$ generates $\mathscr{G}$ as an algebra. For the step $m$ nilpotent Lie algebra $\mathscr{G}$, we have

$$
\begin{array}{clrl}
\mathscr{G}=V_{1} \oplus \cdots \oplus V_{m} ; & {\left[V_{1}, V_{j}\right]} & =V_{j+1}, \\
j=1, \ldots, m-1 ; & {\left[V_{1}, V_{m}\right]} & =\{0\} .
\end{array}
$$

A Lie group is stratified and nilpotent if the corresponding Lie algebra is so.

A Carnot group $\mathbb{G}$ is a stratified simply connected nilpotent Lie group with the Lie algebra $\mathscr{G}$. Let $X_{11}, \ldots, X_{1 n_{1}}$ be a basis of the vector space $V_{1} \subset \mathscr{G}, n_{1}=$ $\operatorname{dim} V_{1}$. From now on, we call $V_{1}$ the horizontal space. Since the vector fields $X_{11}, \ldots, X_{1 n_{1}}$ generate the Lie algebra $\mathscr{G}$, we can choose a basis $X_{i j}, 1 \leq j \leq$ $n_{i}=\operatorname{dim} V_{i}, 1<i \leq m$ of space $V_{i}$, such that $X_{i j} \subset V_{i}$ are commutators of the vector fields $X_{1 j} \subset V_{1}, j=1, \ldots, n_{1}$. We see that the collection $X_{11}, X_{12}, \ldots, X_{1 n_{1}}$ satisfies the Hörmander hypoellipticity condition [17].

It is known [10] that if $\mathbb{C}$ is a simply connected nilpotent Lie group with the Lie algebra $\mathscr{G}$, then the exponential map exp : $\mathscr{G} \rightarrow \mathbb{G}$ is a diffeomorphism from the Lie algebra $\mathscr{G}$ to the Lie group $\mathbb{G}$. Thus, $d x \circ \exp ^{-1}$ is a bi-invariant Haare measure on $\mathbb{G}$, where $d x$ is the Lebesgue measure on $\mathscr{G}$. We can identify the elements $x \in \mathbb{G}$ of the group with the elements $x \in \mathscr{G}$ of the algebra, and thus, with $x \in \mathbb{R}^{N}, N=\sum_{i=1}^{m} \operatorname{dim} V_{i}$, by the exponential map $x=\exp \left(\sum x_{i j} X_{i j}\right)$. The numbers $x=\left(x_{i j}\right), 1 \leq i \leq m, 1 \leq j \leq n_{i}$, are called the coordinates of the point $x$. There is a natural group of dilations, which is defined by the rule $\delta_{r} x=\left(r^{i} x_{i j}\right), 1 \leq i \leq m, 1 \leq j \leq n_{i}$. It is easy to see that $d\left(\delta_{r} x\right)=r^{v} d x$. The quantity $v=\sum_{i=1}^{m} i \cdot n_{i}$ is called the homogeneous dimension of the group $\mathbb{C}$. 
We use the Carnot-Carathéodory metric based on the length of horizontal curves. A piecewise curve $\gamma:[0, b] \rightarrow \mathbb{G}$ is said to be horizontal if its tangent vector $\dot{\gamma}(s)$ belongs to the space $V_{1}$, that is, there exist functions $a_{j}(s)$, such that

$$
\dot{\gamma}(s)=\sum_{j=1}^{n_{1}} a_{j}(s) X_{1 j}(\gamma(s)) .
$$

The result of [6] implies that one can connect two arbitrary points $x, y \in \mathbb{G}$ by a horizontal curve. We fix on $V_{1}$ a nondegenerate quadratic form $\langle\cdot, \cdot\rangle$ so that the vector fields $X_{11}(x), \ldots, X_{1 n_{1}}(x)$ are orthonormal with respect to this form at every $x \in \mathbb{C}$. The length $l(\gamma)$ of a curve $\gamma$ is defined by the formula

$$
l(\gamma)=\int_{0}^{b}\langle\dot{\gamma}(s), \dot{\gamma}(s)\rangle^{1 / 2} d s .
$$

The Carnot-Carathéodory distance $d_{c}(x, y)$ is the infimum of the length over all horizontal curves connecting $x$ and $y \in \mathbb{C}$. Since the quadratic form is left invariant, the Carnot-Carathéodory metric is left invariant as well. The group $\mathbb{G}$ is connected, therefore the metric $d_{c}(x, y)$ is finite (see [36]). We also use a metric, which is generated by a homogeneous norm. The homogeneous norm on $\mathbb{G}$ is, by definition, a continuous nonnegative function $\rho(\cdot)$ on $\mathbb{G}$ such that $\rho(x)=\rho\left(x^{-1}\right), \rho\left(\delta_{r}(x)\right)=r \rho(x)$, and $\rho(x)=0$ if and only if $x=0$, where 0 denotes the identity element of $\mathbb{C}$. The homogeneous norm is not uniquely defined, however, any two homogeneous norms are equivalent. We fix the homogeneous norm $\rho(x)$ of the element $x=\left(x_{1}, \ldots, x_{m}\right), x_{i} \in V_{i}$, which is prescribed by the formula

$$
\rho(x)=\left(\sum_{i=1}^{m}\left|x_{i}\right|^{2 m !}\right)^{1 / 2 m !}, \quad\left|x_{i}\right|=\left(\sum_{j=1}^{n_{i}} x_{i j}^{2}\right)^{1 / 2} .
$$

The homogeneous norm defines the homogeneous metric by the rule $\rho\left(x_{1}, x_{2}\right)$ $=\rho\left(x_{1}^{-1} x_{2}\right)$. The distances $d_{c}\left(x_{1}, x_{2}\right)$ and $\rho\left(x_{1}, x_{2}\right)$ are equivalent.

We let $B(x, r)=\left\{y \in \mathbb{G}: \rho\left(x^{-1} y\right)<r\right\}$ denote the open ball with the center at $x$ and of radius $r>0$ in the metric $\rho$. Note that $B(x, r)$ is the left translation by $x$ of the ball $B(0, r)$, which is the image under $\delta_{r}$ of the "unit ball" $B(0,1)$. The Hausdorff dimension of the metric space $\left(\mathbb{G}, d_{c}\right)$ coincides with its homogeneous dimension $v$. By $|E|$, we denote the measure of the set $E$ : $|E|=\int_{E} d x$. Our normalizing condition is such that the balls of radius one are of measure one; $|B(0,1)|=\int_{B(0,1)} d x=1$. Since the Jacobian determinant of the dilation $\delta_{r}$ is $r^{v}$, we have that $|B(\cdot, r)|=r^{v}$.

The Euclidean space $\mathbb{R}^{n}$ with the standard structure is an example of the Abelian group; the exponential map is the identity and the vector fields $X_{i}=$ $\partial / \partial x_{i}, i=1, \ldots, n$, have only trivial commutative relations and form the basis of the corresponding Lie algebra. 
The simplest example of a non-Abelian group is the Heisenberg group $\mathbb{M}^{n}$. The underlying space is $\mathbb{R}^{2 n+1}$ with the group law of multiplication

$$
(x, t)\left(x^{\prime}, t^{\prime}\right)=\left(x+x^{\prime}, t+t^{\prime}+2 \sum_{i=1}^{n}\left(x_{n+j} x_{j}^{\prime}-x_{j} x_{n+j}^{\prime}\right)\right) .
$$

Here, $x, x^{\prime} \in \mathbb{R}^{2 n}, t, t^{\prime} \in \mathbb{R}$. The Lie algebra $\mathscr{G}$ of the Heisenberg group $\mathbb{M}^{n}$ is generated by the left-invariant vector fields

$$
X_{j}=\frac{\partial}{\partial x_{j}}+2 x_{n+j} \frac{\partial}{\partial t}, \quad X_{n+j}=\frac{\partial}{\partial x_{n+j}}-2 x_{j} \frac{\partial}{\partial t}, \quad 1 \leq j \leq n, T=\frac{\partial}{\partial t} .
$$

Since $\left[X_{j}, X_{n+j}\right]=-4 T$, we have that $X_{j}, X_{n+j},\left[X_{j}, X_{n+j}\right], 1 \leq j \leq n$, generate the tangent space at each point of the group. The vector fields $X_{j}, j=1, \ldots, 2 n$, form a basis of the horizontal vector space $V_{1}$ and $\operatorname{span}\{T\}=V_{2}$. Thus, the Hiesenberg group is a two-step Carnot group. An example of the homogeneous norm is $\rho(x, t)=\left(|x|^{4}+t^{2}\right)^{1 / 4}$. The homogeneous dimension $v$ is equal to $2 n+2$.

Now we define an absolutely continuous function on curves of the horizontal fibration that is formed by the horizontal vector fields $X_{1 j}, j=1, \ldots, n_{1}$ (see terminology, for instance, in [18, 37, 43]).

DEFINITION 2.1. A function $u: \Omega \rightarrow \mathbb{R}, \Omega \subset \mathbb{G}$, is said to be absolutely continuous on lines $(u \in \operatorname{ACL}(\Omega))$ if for any domain $U, \bar{U} \subset \Omega$, and any fibration $\mathscr{X}$ defined by the left-invariant vector fields $X_{1 j}, j=1, \ldots, n_{1}$, the function $u$ is absolutely continuous on $\gamma \cap U$ with respect to the $\mathscr{H}^{1}$-Hausdorff measure for $d \gamma$-almost all curves $\gamma \in \mathscr{X}$.

For the function $u \in \operatorname{ACL}(\Omega)$, the derivatives $X_{1 j} u$ along the vector fields $X_{1 j}, j=1, \ldots, n_{1}$, exist almost everywhere in $\Omega$.

The Sobolev space $W_{p}^{1}(\Omega)\left(L_{p}^{1}(\Omega)\right), 1 \leq p<\infty$, consists of locally summable functions $u: \Omega \rightarrow \mathbb{R}, \Omega \subset \mathbb{G}$, that have distributive derivatives $X_{1 j} u$ along the vector fields $X_{1 j}$ :

$$
\int_{\Omega} X_{1 j} u \varphi d x=-\int_{\Omega} u X_{1 j} \varphi d x, \quad j=1, \ldots, n_{1}
$$

for any test function $\varphi \in C_{0}^{\infty}$ and of finite norm

$$
\begin{gathered}
\left\|u \mid W_{p}^{1}(\Omega)\right\|=\left(\int_{\Omega}|u|^{p} d x\right)^{1 / p}+\left(\int_{\Omega}\left|\nabla_{\mathscr{L}} u\right|^{p} d x\right)^{1 / p}, \\
\left(\left\|u \mid L_{p}^{1}(\Omega)\right\|=\left(\int_{\Omega}\left|\nabla_{\mathscr{L}} u\right|^{p} d x\right)^{1 / p}\right) .
\end{gathered}
$$


The quantity $\nabla_{\mathscr{L}} u=\left(X_{11} u, \ldots, X_{1 n_{1}} u\right)$ is called the subgradient of $u$. For a smooth function, the subgradient is equal to the projection of the usual Riemannian gradient on $V_{1}$. We will say that $u$ belongs to $W_{p, \text { loc }}^{1}(\Omega)\left(L_{p, \text { loc }}(\Omega)\right)$ if for any $U, \bar{U} \subset \Omega$, the function $u$ belongs to $W_{p}^{1}(U)\left(L_{p}(U)\right)$.

DEFINITION 2.2. A mapping $f: \Omega \rightarrow \mathbb{G}, \Omega \subset \mathbb{G}$, belongs to the horizontal Sobolev class $H W_{p, \text { loc }}^{1}(\Omega), 1 \leq p<\infty$, if

(1) $\rho(f(x)) \in L_{p, \text { loc }}(\Omega)$,

(2) the coordinate functions $f_{i j}$ belong to $\operatorname{ACL}(\Omega)$ for all $i$ and $j$,

(3) $f_{1 j} \in W_{p, \text { loc }}^{1}(\Omega)$ for $1 \geq j \geq n_{1}$,

(4) the vector

$$
X_{1 k}(f(x))=\sum_{1 \leq l \leq m, 1 \leq \omega \leq n_{l}} X_{1 k}\left(f_{l \omega}(x)\right) \frac{\partial}{\partial x_{l \omega}}
$$

belongs to the subspace $V_{1}$ for almost all $x \in \Omega$ and $k=1, \ldots, n_{1}$.

In $[37,40]$, the reader can find various definitions of the Sobolev space and their relationships.

The matrix $X_{1 k} f=\left(X_{1 k} f_{i j}\right)$ defines an operator $D_{H} f$ which is called the formal horizontal differential. It maps the horizontal space into the horizontal space $D_{H} f: V_{1} \rightarrow V_{1}$ [27]. A mapping $f \in H W_{p \text {,loc }}^{1}(\Omega)$ which possesses this property is called the (weakly) contact mapping. The norm of the operator $D_{H} f$ is defined by

$$
\left|D_{H} f(x)\right|=\sup _{\xi \in V_{1}, \rho(\xi)=1} \rho\left(D_{H} f(x)(\xi)\right) .
$$

The norm $\left|D_{H} f\right|$ is equivalent to $\left|\nabla_{\mathscr{L}} f\right|=\left(\sum_{i=1}^{n_{1}}\left|X_{1 i} f\right|^{2}\right)^{1 / 2}$. It has been proved in [43] (see also $[26,40]$ ) that the formal horizontal differential $D_{H} f$ generates a homomorphism $D f: \mathscr{G} \rightarrow \mathscr{G}$ of the algebra $\mathscr{G}$, which is called a formal differential. The norm of the operator $D f$ is defined in a usual way as

$$
|D f(x)|=\sup _{\xi \in \mathscr{\xi}, \rho(\xi)=1} \rho(D f(x)(\xi))
$$

and is equal to $\left|D_{H} f\right|$ for almost all $x \in \Omega[40,43]$. The determinant of the matrix $D f(x)$ is denoted by $J(x, f)$ and is called the Jacobian of $f$.

A map is called open if the image of an open set is an open set, and discrete if the preimage of any point consists of isolated points. It is known that for $\mathbb{G}=\mathbb{R}^{n}[30,31]$ and $\mathbb{G}=\mathbb{G}^{n}[8,39]$, inequality (1.1) implies the openness and discreteness of quasiregular mappings. The question about topological properties of quasiregular mappings on an arbitrary Carnot group satisfying only inequality (1.1) is open (see discussion in [9, 13, 39]).

The definition and main results on differentiability on Carnot groups are due to Pansu $[26,27]$. 
DEFINITION 2.3. A mapping $f: \Omega \rightarrow \mathbb{G}$ is called $\mathscr{P}$-differentiable in $x \in \Omega$ if the mapping $\delta_{1 / t}\left(f(x)^{-1} f\left(x \delta_{t} y\right)\right)$ converges locally uniformly to a contact homomorphism $L: \mathbb{G} \rightarrow \mathbb{G}$ as $t \rightarrow 0$.

Some properties of quasiregular mappings are compiled in the next theorem (see [39, 40, 41, 43]).

THeOREM 2.4. A quasiregular mapping $f: \Omega \rightarrow \mathbb{G}, \Omega \subset \mathbb{G}$, possesses the following properties:

(1) $\mathscr{P}$-differentiable almost everywhere in $\Omega$;

(2) Lusin's $\mathcal{N}$-property: $|A|=0$ implies $|f(A)|=0$;

(3) $\mathcal{N}^{-1}$-property: $|A|=0$ implies $\left|f^{-1}(A)\right|=0$;

(4) $J(x, f)>0$ almost everywhere in $\Omega$;

(5) $\left|B_{f}\right|=\left|f\left(B_{f}\right)\right|=0$.

We use the notation $U(x, s)$ for the $x$-component of the set $f^{-1}(B(f(x), s))$. If $x \in \Omega, 0<r<\rho(x, \partial \Omega)$, and $0<s<\rho(f(x), \partial f(\Omega))$, then

$$
\begin{array}{cc}
l(x, r)=\inf _{\rho(x, z)=r} \rho(f(z), f(x)), & L(x, r)=\sup _{\rho(x, z)=r} \rho(f(z), f(x)), \\
l^{\star}(x, s)=\inf _{y \in \partial U(x, f, s)} \rho(x, y), & L^{\star}(x, s)=\sup _{y \in \partial U(x, f, s)} \rho(x, y) .
\end{array}
$$

Making use of the method of the paper [22], we can show that if $C \subset \Omega$ is a compact, then the function $(x, s) \mapsto l^{\star}(x, s)$ is continuous and $(x, s) \mapsto L^{\star}(x, s)$ is lower semicontinuous on the set $C \times(0, t)$.

For the mapping $f: \Omega \rightarrow \mathbb{G}$, we denote by

$$
H(x, f)=\limsup _{r \rightarrow 0} \frac{L(x, r)}{l(x, r)}, \quad H^{\star}(x, f)=\limsup _{s \rightarrow 0} \frac{L^{\star}(x, s)}{l^{\star}(x, s)}
$$

the linear distortion and the inverse linear distortion of $f$ at $x \in \Omega$, respectively.

A subdomain $D \subset \Omega$ is called a normal domain under a mapping $f: \Omega \rightarrow \mathbb{G}$ if $f(\partial D)=\partial f(D)$. A neighborhood $U \subset \Omega$ of a point $x \in \Omega$ is called a normal neighborhood under a mapping $f: \Omega \rightarrow \mathbb{G}$ if it is a normal domain and $U \cap$ $f^{-1}(f(x))=\{x\}$.

The next topological facts can be proved for a rather broad class of metric spaces. For the Euclidean space $\mathbb{R}^{n}$, the proof can be found in [14, 22, 32]. By the symbol $\mathbb{C} D$ we will denote the complement to the domain $D$.

LEMMA 2.5. Let $f: \Omega \rightarrow \mathbb{G}$ be a continuous open discrete and sense preserving mapping. Then for a point $x \in \Omega$, there exists $\sigma_{x}>0$ such that for any $0<s \leq$ $\sigma_{x}$, the following conditions hold:

(1) $U(x, s)$ is the normal neighborhood of $x$;

(2) $U(x, s)=U\left(x, \sigma_{x}\right) \cap f^{-1}(B(f(x), s))$;

(3) if $s<\sigma_{x}$, then $\partial U(x, s)=U\left(x, \sigma_{x}\right) \cap f^{-1}(S(f(x), s))$; 
(4) the sets $\mathbb{C} U(x, s)$ and $\mathbb{C} \bar{U}(x, s)$ are connected;

(5) $U(x, s) \backslash \bar{U}(x, t)$ is a ring-type domain for $0<t<s \leq \sigma_{x}$;

(6) $l^{\star}(x, L(x, r))=L^{\star}(x, l(x, r))=r$ for $r \in\left(0, l^{\star}\left(x, \sigma_{x}\right)\right)$.

If $U=U(x, s)$ is a normal neighborhood of $x$, then the degree $\mu(y, f, U)$ of continuous open discrete mapping $f$ with respect to $y=f(x)$ is constant for any point $z \in f(U)$ and is denoted by $\mu(f, U)$. The definition of the mapping degree can be found in [30]. In the same work, it was proved that for a compact subdomain $G \subset U$, the degree $\mu(y, f, G), y=f(x), x \in U$, has the same value as for $U$, which is called the local index $i(x, f)$ of the point $x \in U$. The index $i(x, f)$ is equal to 1 if and only if $x$ is not a branch point.

We define the quantity

$$
N(f, A)=\sup _{y \in \mathbb{G}} N(y, f, A)=\sup _{y \in \mathbb{G}} \operatorname{card}\left\{f^{-1}(y) \cap A\right\}
$$

and call it a multiplicity function. If a set $A$ is such that $\bar{A}$ is compact and $\bar{A} \subset \Omega$, then the discreteness implies that $N(f, A)$ is finite.

Some relations between the multiplicity function, the mapping degree, and the local index are expressed in the following lemma. Its proof for the Carnot group can be obtained as in [21, Lemma 2.12].

LEMMA 2.6. Let $f: \Omega \rightarrow \mathbb{G}$ be a continuous open discrete and sense preserving mapping. If $U \subset \Omega$ is a normal domain for $f$, then

(1) $N(f, U)=\mu(y, f, U)=\mu(f, U)$ for all $y \in f(U)$;

(2) $\mu(y, f, U)=\sum_{j=1}^{k} i\left(x_{j}, f\right)$ for all $y \in f(U)$, where $k=N(y, f, U)$ and $\left\{x_{1}, \ldots, x_{k}\right\}=f^{-1}(y) \cap U$

(3) $i(x, f)=N(f, U)$ if and only if $U$ is the normal neighborhood of a point $x \in U$.

3. Estimates of the measure of the image of the branch set. The value

$$
\mathscr{H}^{\alpha}(A)=\liminf _{\varepsilon \rightarrow 0}\left\{\sum_{i} \operatorname{diam}^{\alpha}\left(B_{i}\right) \mid A \subset \cup B_{i} \operatorname{diam} B_{i}<\varepsilon\right\}
$$

is called the Hausdorff $\alpha$-measure. We denote by $\operatorname{diam}_{\rho}\left(B_{i}\right)$ the diameter of the set $B_{i}$ with respect to the homogeneous metric, and by $\operatorname{diam}_{\|\cdot\|}\left(B_{i}\right)$ the diameter under the Euclidean metric. The Hausdorff $\alpha$-measure with respect to these different metrics we note by $\mathscr{H}_{\rho}^{\alpha}$ and $\mathscr{H}_{\|\cdot\|}^{\alpha}$, respectively.

For the beginning, we give a simple proposition on the positiveness of the Hausdorff $(N-2)$-measure of the image of the branch set of quasiregular mappings on Carnot groups.

LEMMA 3.1. Let $f: \Omega \rightarrow \mathbb{G}$ be a quasiregular mapping on a Carnot group with $B_{f} \neq \varnothing$. Then $\mathscr{H}_{\rho}^{N-2}\left(f\left(B_{f}\right)\right)>0, N=\sum_{i=1}^{m} \operatorname{dim} V_{i}$. 
Proof. From [23], it follows that for an open discrete map in the Euclidean space $\mathbb{R}^{N}$, the following estimate $\mathscr{H}_{\|\cdot\|}^{N-2}\left(f\left(B_{f}\right)\right)>0$ holds. We cover $f\left(B_{f}\right)$ by open balls $\left\{B_{i}\right\}, i=1,2, \ldots$. We have the inequality $\|\cdot\| \leq \rho$ by the definition of the homogeneous metric $\rho$. Then

$$
\begin{aligned}
\mathscr{H}_{\rho}^{N-2}\left(f\left(B_{f}\right)\right) & =\liminf _{\varepsilon \rightarrow 0} \sum_{i} \operatorname{diam}_{\rho}^{N-2}\left(B_{i}\right) \\
& \geq \liminf _{\varepsilon \rightarrow 0} \sum_{i} \operatorname{diam}_{\|\cdot\|}^{N-2}\left(B_{i}\right)=H_{\|\cdot\|}^{N-2}\left(f\left(B_{f}\right)\right)>0 .
\end{aligned}
$$

Now, we give more precise estimates for the Hausdorff measure of the image of the branch set under quasiregular mappings on Carnot groups. First of all, we describe a set $P_{z}(E)$. We need this construction in the following lemma. Let $z, \omega$ be disjoint points on the group $\mathbb{G}$. We denote by $\sigma_{\omega}^{z}(t)=z \delta_{t}(\omega)$ the oneparametric curve which starts from the point $z$ and passes through the point $\omega$. We let $E$ be a set on $\mathbb{G}$ and let $z \notin E$. The collection of the one-parametric curves $\sigma_{\omega}^{z}(t), \omega \in E$, we denote by $P_{z}(E)$.

LEMMA 3.2. Let $E \subset \mathbb{G}$ be a set such that $\mathscr{H}_{\rho}^{\alpha}(E)=0$ for any $\alpha>0$. Then $\mathcal{H}_{\rho}^{\alpha+m}\left(P_{z}(E)\right)=0$ for $z \notin E$. Here $m$ is the index of the last vector space in the graduated Lie algebra $\mathscr{G}$ of $\mathbb{G}$.

Proof. Since the Haar measure is invariant with respect to the left translation, we can assume that $z=0$, and from now on we omit the letter $z$. Moreover, up to the end of the proof, the notations $B_{\rho}(x, r), S_{\rho}(x, r)$ mean the ball and the sphere, respectively, under the homogeneous metric $\rho$, and $B_{\|\cdot\|}(x, r)$, $S_{\|\cdot\|}(x, r)$ mean the ball and the sphere with respect to the Euclidean metric.

We will use the notations $R_{k}=\{x \in \mathbb{G}: 1 / k \leq \rho(x) \leq k, k \in \mathbb{N}\}, E_{k}=E \cap R_{k}$, and $P_{k}=P\left(E_{k}\right) \cap R_{k}$. Since $P(E)=\bigcup_{k=1}^{\infty} P_{k}$, the lemma will be proved if we establish the equality $\mathscr{H}_{\rho}^{\alpha+m}\left(P_{k}\right)=0$ for any $k \in \mathbb{N}$. Since $\mathscr{H}_{\rho}^{\alpha}\left(E_{k}\right)=0$ for any $k$, we cover $E_{k}$ by a system of balls $B_{\rho}\left(x_{i}, r_{i}\right)$, such that

(1) $x_{i} \in E_{k}$,

(2) $r_{i}<1 / 4 k^{2}$,

(3) $\sum_{i} r_{i}^{\alpha}<\varepsilon$, for any $\varepsilon \in(0,1)$.

We can assume that the intersection $E_{k} \cap B_{\rho}\left(x_{i}, r_{i}\right)$ is not empty for any index $i$, otherwise we omit such balls. Let $H_{i}$ denote the set $P\left(B_{\rho}\left(x_{i}, r_{i}\right)\right) \cap R_{k}$. Then $P_{k} \subset \bigcup_{i} H_{i}$. We fix $i \in \mathbb{N}$. Since the homogeneous norm of the point $\bar{x}_{i}$ satisfies the equalities

$$
\rho\left(\bar{x}_{i}\right)=\rho\left(\delta_{k / \rho\left(x_{i}\right)}\left(x_{i}\right)\right)=\frac{k}{\rho\left(x_{i}\right)} \rho\left(x_{i}\right)=k,
$$

the point $x_{i}$ is mapped onto $\bar{x}_{i} \in S_{\rho}(0, k)$ under the dilation $\delta_{k / \rho\left(x_{i}\right)}\left(x_{i}\right)$. Similarly, $\delta_{1 / k \rho\left(x_{i}\right)}\left(x_{i}\right)=\underline{x}_{i}, \underline{x}_{i} \in S_{\rho}(0,1 / k)$. Thus, if

$$
t \in J_{i}=\left[\frac{1}{k \rho\left(x_{i}\right)}, \frac{k}{\rho\left(x_{i}\right)}\right] \subset\left[\frac{1}{k^{2}}, k^{2}\right],
$$


then a point $x \in \sigma_{x_{i}}(t)$ is located between the points $\underline{x}_{i} \in S_{\rho}(0,1 / k)$ and $\bar{x}_{i} \in$ $S_{\rho}(0, k)$.

We want to estimate the Euclidean length of the part of the curve $\sigma_{x_{i}}(t)$ which lies in $R_{k}$. The parametric equation of $\sigma_{x_{i}}(t)$ in the Euclidean coordinates of $\mathbb{R}^{N}$ is of the form

$$
\sigma_{x_{i}}^{(1)}(t)=\frac{t}{\rho\left(x_{i}\right)} x_{i}^{(1)}, \ldots, \sigma_{x_{i}}^{(m)}(t)=\left(\frac{t}{\rho\left(x_{i}\right)}\right)^{m} x_{i}^{(m)},
$$

where $x_{i}^{(l)}=\left(x_{i}^{(l 1)}, \ldots, x_{i}^{\left(\ln _{l}\right)}\right)$ are the coordinates of the point $x_{i}$ in the vector space $V_{l}$. The Euclidean length $l\left(\sigma_{x_{i}}^{k}\right)$ of $\sigma_{x_{i}}^{k}=\sigma_{x_{i}}(t) \cap R_{k}$ is given by the integral

$$
\begin{aligned}
l\left(\sigma_{x_{i}}^{k}\right) & =\int_{1 / k}^{k}\left(\left\|\frac{x_{i}^{(1)}}{\rho\left(x_{i}\right)}\right\|^{2}+\left\|\frac{2 t x_{i}^{(2)}}{\rho^{2}\left(x_{i}\right)}\right\|^{2}+\cdots+\left\|\frac{m t^{m-1} x_{i}^{(m)}}{\rho^{m}\left(x_{i}\right)}\right\|^{2}\right)^{1 / 2} d t \\
& \leq \int_{0}^{k}\left(1+(2 t)^{2}+\cdots+\left(m t^{m-1}\right)^{2}\right)^{1 / 2} d t \leq N m^{3 / 2} k^{m}=L
\end{aligned}
$$

and does not depend on $i \in \mathbb{N}$. Here we have used the estimates $\left\|x_{i}^{(l)}\right\| / \rho^{l}\left(x_{i}\right) \leq$ $1, l=1, \ldots, m$.

If $x \in B_{\rho}\left(x_{i}, r_{i}\right)$, then

$$
\rho\left(\delta_{k / \rho\left(x_{i}\right)}\left(x_{i}\right), \delta_{k / \rho\left(x_{i}\right)}(x)\right)=\frac{k}{\rho\left(x_{i}\right)} \rho\left(x_{i}, x\right)=\frac{k}{\rho\left(x_{i}\right)} r_{i} \leq k^{2} r_{i}<1 .
$$

We divide the arc $\sigma_{x_{i}}^{k}$ into parts of equal Euclidean length which does not exceed $C \cdot\left(k^{2} r_{i}\right)^{m}<1$ by points $\underline{x}_{i}=s_{i 0}, s_{i 1}, \ldots, s_{i N_{i}}=\bar{x}_{i}$. Here $C$ is a constant from [25, Proposition 1.1], which depends on $k$. Let $N_{i}$ be the biggest positive integer such that $N_{i} \leq L /\left(C \cdot\left(k^{2} r_{i}\right)^{m}\right)+1$. To this partition of the curve corresponds the following partition of the interval $J_{i}$ :

$$
\frac{1}{k^{2}} \leq \frac{1}{k \rho\left(x_{i}\right)}=T_{0} \leq T_{1} \leq \cdots \leq T_{N_{i}}=\frac{k}{\rho\left(x_{i}\right)} \leq k^{2}, \quad \delta_{T_{j}}\left(x_{i}\right)=s_{i j} .
$$

The collection of the balls $\left\{B_{\|\cdot\|}\left(s_{i j}, C \cdot\left(k^{2} r_{i}\right)^{m}\right), j=0, \ldots, N_{i}\right\}$ covers the curve $\sigma_{x_{i}}^{k}$. We claim that

(1) $\sigma_{x_{i}}^{k} \subset \bigcup_{j=1}^{N_{i}} B_{\rho}\left(s_{i j}, k^{2} r_{i}\right), N_{i} \leq L / C \cdot\left(k^{2} r_{i}\right)^{m}+1$,

(2) $H_{i} \subset \bigcup_{j=1}^{N_{i}} B_{\rho}\left(s_{i j}, 2 k^{2} r_{i}\right), N_{i} \leq L / C \cdot\left(k^{2} r_{i}\right)^{m}+1$.

The first assertion follows (see [25, Proposition 1.1]) from the inclusions $B_{\|\cdot\|}\left(s_{i j}, C\left(k^{2} r_{i}\right)^{m}\right) \subset B_{\rho}\left(s_{i j}, k^{2} r_{i}\right)$. We show the second one. If $y \in H_{i}$, then there is a point $x \in B_{\rho}\left(x_{i}, r_{i}\right)$ such that

$$
\begin{gathered}
y=\delta_{t}(x), \quad \tilde{s}=\delta_{t}\left(x_{i}\right)=\sigma_{x_{i}}(t), \\
\rho(\tilde{s}, y)=\rho\left(\delta_{t}\left(x_{i}\right), \delta_{t}(x)\right)=t \rho\left(x_{i}, x\right)=t r_{i} \leq k^{2} r_{i},
\end{gathered}
$$


for some $t \in\left[T_{j-1}, T_{j}\right]$. Furthermore, the first statement implies that the point $\tilde{s}$ belongs to $B_{\rho}\left(s_{i j}, k^{2} r_{i}\right)$. From this and from the triangle inequality, we have $\rho\left(s_{i j}, y\right) \leq \rho\left(s_{i j}, \tilde{s}\right)+\rho(\tilde{s}, y) \leq 2 k^{2} r_{i}$. Thus $y \in B_{\rho}\left(s_{i j}, 2 k^{2} r_{i}\right)$ and the second assertion is proved.

From the statement above, we obtain that the countable collection of the balls with respect to the homogeneous metric $\left\{B_{\rho}\left(s_{i j}, 2 k^{2} r_{i}\right)\right\}, j=1, \ldots, N_{i}$, $i \in \mathbb{N}$, covers the set $P_{k}$. Finally, we have

$$
\begin{aligned}
\mathscr{H}_{\rho}^{\alpha+m}\left(P_{k}\right) & \leq \sum_{i} \sum_{j=1}^{N_{i}}\left(\operatorname{diam}_{\rho} B_{\rho}\left(s_{i j}, 2 k^{2} r_{i}\right)\right)^{\alpha+m}=\sum_{i} \sum_{j=1}^{N_{i}}\left(4 k^{2} r_{i}\right)^{\alpha+m} \\
& \leq \sum_{i} \frac{1+L}{C \cdot\left(k^{2} r_{i}\right)^{m}} 4^{\alpha+m}\left(k^{2} r_{i}\right)^{\alpha+m}=\frac{1+L}{C} 4^{\alpha+m} k^{2 \alpha} \sum_{i} r_{i}^{\alpha} \\
& \leq M(m, k) \varepsilon .
\end{aligned}
$$

Since $\varepsilon>0$ is arbitrary, we get $\mathscr{H}_{\rho}^{\alpha+m}\left(P_{k}\right)=0$.

The proof of the next theorem is based on the idea of [11, Theorem 1] and [30, Theorem 10.3].

THEOREM 3.3. Let $\Omega \subset \mathbb{G}$ be an open connected set, and let $f: \Omega \rightarrow \mathbb{G}$ be a nonconstant quasiregular mapping such that the set of branch points $B_{f}$ is not empty. Then

$$
\mathscr{H}_{\rho}^{\nu-(m+1)}\left(f\left(B_{f}\right)\right)>0 .
$$

Here $m$ is the index of the last vector space in the graduated Lie algebra 9 of $\mathbb{G}$.

Proof. We suppose, contrary to our claim, that $\mathscr{H}_{\rho}^{\nu-(m+1)}\left(f\left(B_{f}\right)\right)=0$. We choose $x_{0} \in B_{f}$ and we put $y_{0}=f\left(x_{0}\right)$. Let $\sigma_{x_{0}}>0$ be the number from Lemma 2.5 such that $\bar{B}\left(x_{0}, \sigma_{x_{0}}\right) \subset \Omega$, and let $l_{0}=(1 / 2) l\left(x_{0}, \sigma_{x_{0}}\right)$. Then $U=$ $U\left(x_{0}, l_{0}\right)$ is the normal neighborhood of $x_{0}$, and the inclusions $B\left(y_{0}, l_{0}\right) \subset$ $f\left(B\left(x_{0}, \sigma_{x_{0}}\right)\right), \bar{U} \subset B\left(x_{0}, \sigma_{x_{0}}\right)$, and $f(U) \subset B\left(y_{0}, l_{0}\right)$ hold.

We show that $f\left(B_{f} \cap U\right)$ is a closed set in $B\left(y_{0}, l_{0}\right)$. In fact, if $z \in B\left(y_{0}, l_{0}\right)$ is an adherent point for $f\left(B_{f} \cap U\right)$, then there exists a sequence $\left\{t_{k}\right\} \in B_{f} \cap U$, $k=1,2, \ldots$, such that $f\left(t_{k}\right) \rightarrow z$. We may assume that $\left\{t_{k}\right\}$ converges to $t_{0} \in \bar{U}$. Since $B_{f}$ is closed in $\Omega$ and $\bar{U} \subset \Omega$, then $t_{0} \in B_{f}$ and we have $f\left(t_{0}\right)=z$ by the continuity of $f$. The ball $B\left(y_{0}, l_{0}\right)$ does not contain points of the boundary $\partial U$, therefore $t_{0} \in U$. Consequently, $z \in f\left(B_{f} \cap U\right)$.

We set $E=f\left(B_{f} \cap U\right)$. By the assumption $\mathscr{H}_{\rho}^{\nu-(m+1)}(E)=0$, there is a point $z \in B\left(y_{0}, l_{0}\right)$ such that $z \notin E$. We construct the set $P_{z}(E)$. Lemma 3.2 implies that $\mathscr{H}_{\rho}^{\nu-1}\left(P_{z}(E)\right)=0$. The set $E$ is closed in $B\left(y_{0}, l_{0}\right)$, and the intersection $B\left(y_{0}, l_{0}\right) \cap P_{z}(E)$ is also closed. Thus $H=B\left(y_{0}, l_{0}\right) \backslash P_{z}(E)$ is open. We show that the set $H$ is simple connected. Really, since $z \notin E$, any closed curve $\gamma$ can 
be shrunk into the point $z$ along the one-parametric curves $\sigma_{\omega}^{z}(t)$, which are contained in $H$ and connect $z$ with the points $\omega \in \gamma$.

Let $W \subset U$ be a connected component of $f^{-1}(H)$, which is contained in $U$. Then $\bar{W} \subset B\left(x_{0}, \sigma_{x_{0}}\right), f(W)=H, f(\partial W) \subset \partial H$, and the restriction $\left.f\right|_{W}$ is a quasiconformal mapping [42, 44].

If $W_{1}, W_{2}, \ldots, W_{k}$ are connected components of $f^{-1}(H) \cap U$ and $f_{k}^{-1}$ is the inverse mapping to the restriction $\left.f\right|_{W_{k}}$, then $f_{k}^{-1}$ is a quasiconformal mapping on $H=B\left(y_{0}, l_{0}\right) \backslash P_{z}(E)$. Since we have $\mathscr{H}_{\rho}^{\nu-1}\left(P_{z}(E)\right)=0$, [37, Theorem 8] implies that $f_{k}^{-1}$ is extended to a quasiconformal mapping $\tilde{f}_{k}^{-1}: B\left(y_{0}, l_{0}\right) \rightarrow U$. The set $W_{0}=\tilde{f}_{k}^{-1}\left(B\left(y_{0}, l_{0}\right)\right) \subset U$ is open and closed in $U, U$ is connected, thus $W_{0}=U$. Therefore, $f: U \rightarrow \mathbb{G}$ is a homeomorphism. This contradicts the fact that the set $U$ contains branch points of $f$.

4. Bounds for the local index. We mention some auxiliary definitions and results. Let $U$ be an open set on the Carnot group $\mathbb{G}$ and let $V$ be a compact in $U$. The ordered pair $(U, V)$ is called a condenser.

DEFINITION 4.1. For $p \in[1, \infty)$, the quantity

$$
\operatorname{cap}_{p}(U, V)=\inf \left(\int_{U}\left|\nabla_{\mathscr{L}} v\right|^{p} d x\right)^{1 / p}
$$

is called the $p$-capacity of the condenser $(U, V)$. The infimum is taken over all nonnegative functions $v \in C_{0}^{\infty}(U)$ such that $\left.v\right|_{V} \geq 1$.

The properties of the $p$-capacity in the spaces with the geometry of vector fields satisfying the Hörmander hypoellipticity condition can be found in [4, 5]. If $U$ is an open ball and $V$ is a closed ball with the same center and of smaller radius, then such a condenser $(U, V)$ is called the ring condenser.

LEMmA 4.2. Let $\Omega$ be a domain in $\mathbb{G}$. Then for the condenser $(B(x, R), \bar{B}(x$, $r)), r<R$, there are constants $\theta_{1}, \theta_{2}$, independent of $r$ and $R$, such that

$$
\theta_{1}\left(\ln \frac{R}{r}\right)^{1-v} \leq \operatorname{cap}_{v}(B(x, R), \bar{B}(x, r)) \leq \theta_{2}\left(\ln \frac{R}{r}\right)^{1-v}
$$

The proof of Lemma 4.2 follows from [5, Theorems 6.6 and 6.9].

In the next lemma, some properties of the $v$-capacity of some condensers are given. They have been established in [42] for more general mappings with bounded $s$-distortion. We formulate them for the case $s=v$.

LEMMA 4.3. Let $f: \Omega \rightarrow \mathbb{G}$ be a quasiregular mapping. If $U \subset \Omega$ is a normal domain such that $N(f, U)<\infty$, then

$$
\operatorname{cap}_{v}(U, V) \leq K_{O}(f) N(f, U) \operatorname{cap}_{v}(f(U), f(V)) .
$$


If $U=U\left(x, r_{0}\right)$ is a normal neighborhood of $x \in \Omega$, then, for $0<r<r_{0}$,

$$
\operatorname{cap}_{v}\left(f\left(U\left(x, r_{0}\right)\right), f(\bar{U}(x, r))\right) \leq \frac{K_{I}(f)}{i(x, f)} \operatorname{cap}_{v}\left(U\left(x, r_{0}\right), \bar{U}(x, r)\right) .
$$

We show that the inverse linear distortion $H^{\star}(x, f)$ of the quasiregular mapping $f$ is bounded above, and the estimate does not depend on $x \in \Omega$.

LEMmA 4.4. Let $f: \Omega \rightarrow \mathbb{G}$ be a quasiregular mapping and $K(f)$ be a coefficient of quasiregularity. Then

$$
H^{\star}(x, f) \leq C^{\star}(\nu, K(f)),
$$

where the constant $C^{\star}$ depends on the homogeneous dimension $v$ and on $K(f)$.

Proof. Let $x \in \Omega$ and let $\sigma_{x}>0$ be a number defined in Lemma 2.5. Let $r>0$ be such that $L^{\star}(x, r)<l^{\star}\left(x, \sigma_{x}\right)$. We claim that $L^{\star}(x, r) / l^{\star}(x, r) \leq$ $C^{\star}(\nu, K(f))$. If we denote $l=l\left(x, l^{\star}(x, r)\right), L=L\left(x, L^{\star}(x, r)\right)$, then we get the inequality $l \leq r \leq L$. Since $L^{\star}(x, r)<l^{\star}\left(x, \sigma_{x}\right)$, we have $L<\sigma_{x}$. The boundaries $\partial U(x, l), \partial U(x, r)$ of the normal neighborhood intersect the sphere $S\left(x, l^{\star}(x, r)\right)$. Besides, $\partial U(x, r)$ and $\partial U(x, L)$ intersect $S\left(x, L^{\star}(x, r)\right)$. Thus, [5, Theorem 6.6] implies that the capacities of the condensers $(U(x, r), \bar{U}(x, l))$, $(U(x, L), \bar{U}(x, r))$ are strictly positive and bounded away from zero by a constant $a_{v}$. Since $U(x, r), U(x, L)$ are normal neighborhoods of $x$, then $N(f, U(x$, $r))=N(f, U(x, L))=i(x, f)$. Lemmas 4.2 and 4.3 imply the inequalities

$$
\begin{aligned}
a_{v} & \leq \operatorname{cap}_{v}(U(x, r), \bar{U}(x, l)) \\
& \leq K_{O}(f) i(x, f) \operatorname{cap}_{v}(B(f(x), r), \bar{B}(f(x), l)) \\
& \leq K_{O}(f) i(x, f) \theta_{2}\left(\ln \frac{r}{l}\right)^{1-v}, \\
a_{v} & \leq \operatorname{cap}_{v}(U(x, L), \bar{U}(x, r)) \\
& \leq K_{O}(f) i(x, f) \operatorname{cap}_{v}(B(f(x), L), \bar{B}(f(x), r)) \\
& \leq K_{O}(f) i(x, f) \theta_{2}\left(\ln \frac{L}{r}\right)^{1-v} .
\end{aligned}
$$

From the inclusions $U(x, L) \supset B\left(x, L^{\star}(x, r)\right), U(x, l) \subset B\left(x, l^{\star}(x, r)\right)$, and from Lemma 4.3, we deduce that

$$
\begin{aligned}
\theta_{2}\left(\ln \frac{L^{\star}(x, r)}{l^{\star}(x, r)}\right)^{1-v} & \geq \operatorname{cap}_{v}\left(B\left(x, L^{\star}(x, r)\right), \bar{B}\left(x, l^{\star}(x, r)\right)\right) \\
& \geq \operatorname{cap}_{v}(U(x, L), \bar{U}(x, l)) \\
& \geq \frac{i(x, f)}{K_{I}(f)} \operatorname{cap}_{v}(f(U(x, L)), f(\bar{U}(x, l))) \\
& =\frac{i(x, f) \theta_{1}}{K_{I}(f)}\left(\ln \frac{L}{l}\right)^{1-v} .
\end{aligned}
$$


Making use of the estimates (4.6), we derive

$$
\begin{aligned}
\left(\ln \frac{L}{l}\right)^{v-1} & =\left(\ln \frac{L}{r}+\ln \frac{r}{l}\right)^{v-1} \leq 2^{v-2}\left(\left(\ln \frac{L}{r}\right)^{v-1}+\left(\ln \frac{r}{l}\right)^{v-1}\right) \\
& \leq 2^{v-1} K_{O}(f) i(x, f) \theta_{2} a_{v}^{-1} .
\end{aligned}
$$

Using the inequality (4.7), we obtain

$$
\left(\ln \frac{L^{\star}(x, r)}{l^{\star}(x, r)}\right)^{v-1} \leq \frac{2^{v-1} K_{O}(f) K_{I}(f) \theta_{2}^{2}}{a_{\nu} \theta_{1}} .
$$

Thus,

$$
\frac{L^{\star}(x, r)}{l^{\star}(x, r)} \leq \exp \left(2\left(\frac{K_{O}(f) K_{I}(f) \theta_{2}^{2}}{a_{v} \theta_{1}}\right)^{1 /(v-1)}\right)=C^{\star}(\nu, K(f)) .
$$

Letting $r \rightarrow 0$, we get the required result.

THEOREM 4.5. Let $f: \Omega \rightarrow \mathbb{G}, \Omega \subset \mathbb{G}$, be a quasiregular mapping, and let $F \subset B_{f}$ be a compact set, such that $\mathcal{H}_{\rho}^{\alpha}(f(F))>0$ for some $\alpha$. Then

$$
\inf _{x \in F} i(x, f)<K_{I}(f) \frac{\theta_{2}}{\theta_{1}}\left(\frac{v}{\alpha}\right)^{v-1}
$$

Proof. Lemma 4.4, the continuity of the function $L^{\star}(x, r)$, and the lower continuity of $l^{\star}(x, r)$ imply that there is a number $i_{0} \in \mathbb{N}$ such that the sets

$$
F_{i}=\left\{x \in F, s \in\left(0, \frac{1}{i}\right): \frac{L^{\star}(x, s)}{l^{\star}(x, s)} \leq C^{\star}+1\right\}
$$

are compact for all $i \geq i_{0}$. Since $F=\bigcup_{i} F_{i}$, we can find a number $i \geq i_{0}$, such that $\mathscr{H}_{\rho}^{\alpha}\left(f\left(F_{i}\right)\right)>0$. We represent $F_{i}$ as a union of the sets $F_{i, j}=\left\{x \in F_{i}\right.$ : $i(x, f)=j\}, j=1,2, \ldots$ We fix $j$ such that $\mathcal{H}_{\rho}^{\alpha}\left(f\left(F_{i, j}\right)\right)>0$. Now we choose a point $x_{0} \in F_{i, j}$ and a number $\sigma$ satisfying the properties

(1) $0<\sigma<1 / i$,

(2) $U\left(x_{0}, \sigma\right)$ is a normal neighborhood of $x_{0}$,

(3) $\mathscr{H}_{\rho}^{\alpha}\left(f\left(F_{i, j} \cap U\left(x_{0}, s\right)\right)\right)>0$ for $0<s \leq \sigma$.

For some $0<s \leq \sigma$, we may select $u>0$ such that the inclusions

$$
\bar{U}\left(x_{0}, s\right) \subset B\left(x_{0}, u\right) \subset B\left(x_{0}, 2 u\right) \subset U\left(x_{0}, \sigma\right)
$$

hold. We suppose that inequality (4.11) is false. If we established the equality $\mathscr{H}_{\rho}^{\alpha}\left(f\left(F_{i, j} \cap U\left(x_{0}, s\right)\right)\right)=0$, we would get the contradiction with property (3). Since $\left|B_{f}\right|=0$, we can cover $F_{i, j} \cap U\left(x_{0}, s\right)$ by the balls $B\left(x_{k}, r_{k}\right), k=1,2, \ldots$ such that

(P1) $x_{k} \in F_{i, j} \cap U\left(x_{0}, s\right)$,

(P2) $\sum_{k} r_{k}^{v}<\varepsilon$ for any $\varepsilon \in(0,1)$. 
From the equality $i\left(x_{k}, f\right)=i\left(x_{0}, f\right)$ and Lemma 2.6, we deduce that $U\left(x_{k}\right.$, $\left.L_{k}\right), L_{k}=L\left(x_{k}, r_{k}\right)$, is the normal neighborhood of $x_{k}$. Our supposition and Lemma 4.3 imply

$$
\begin{aligned}
\operatorname{cap}_{v} & \left(f\left(U\left(x_{0}, \sigma\right)\right), f\left(\bar{U}\left(x_{k}, L_{k}\right)\right)\right) \\
\leq & \frac{K_{I}(f)}{i\left(x_{0}, f\right)} \operatorname{cap}_{v}\left(U\left(x_{0}, \sigma\right), \bar{U}\left(x_{k}, L_{k}\right)\right) \\
\leq & \left(\frac{\alpha}{v}\right)^{v-1} \frac{\theta_{1}}{\theta_{2}} \operatorname{cap}_{v}\left(U\left(x_{0}, \sigma\right), \bar{U}\left(x_{k}, L_{k}\right)\right) .
\end{aligned}
$$

The inclusions $f\left(U\left(x_{0}, \sigma\right)\right) \subset B\left(f\left(x_{k}\right), 2 \sigma\right)$ and $\bar{B}\left(f\left(x_{k}\right), L_{k}\right) \subset f\left(\bar{U}\left(x_{k}, L_{k}\right)\right)$, in sequel, give us the inequality

$$
\begin{aligned}
& \operatorname{cap}_{v}(\left.B\left(f\left(x_{k}\right), 2 \sigma\right), \bar{B}\left(f\left(x_{k}\right), L_{k}\right)\right) \\
& \leq \operatorname{cap}_{v}\left(f\left(U\left(x_{0}, \sigma\right)\right), f\left(\bar{U}\left(x_{k}, L_{k}\right)\right)\right) .
\end{aligned}
$$

Analogously, from the insertions $B\left(x_{k}, u\right) \subset U\left(x_{0}, \sigma\right)$ and $B\left(x_{k}, L_{k}^{\star}\right) \supset U\left(x_{k}\right.$, $\left.L_{k}\right), L_{k}^{\star}=L^{\star}\left(x_{k}, L_{k}\right)$, we obtain

$$
\operatorname{cap}_{v}\left(U\left(x_{0}, \sigma\right), \bar{U}\left(x_{k}, L_{k}\right)\right) \leq \operatorname{cap}_{v}\left(B\left(x_{k}, u\right), \bar{B}\left(x_{k}, L_{k}^{\star}\right)\right) .
$$

By inequalities (4.15), (4.16), and Lemma 4.2, we have

$$
\begin{aligned}
\theta_{1}\left(\ln \frac{2 \sigma}{L_{k}}\right)^{1-v} & \leq \operatorname{cap}_{v}\left(f\left(U\left(x_{0}, \sigma\right)\right), f\left(\bar{U}\left(x_{k}, L_{k}\right)\right)\right) \\
& \leq\left(\frac{\alpha}{v}\right)^{v-1} \frac{\theta_{1}}{\theta_{2}} \operatorname{cap}_{v}\left(U\left(x_{0}, \sigma\right), \bar{U}\left(x_{k}, L_{k}\right)\right) \\
& \leq\left(\frac{\alpha}{v}\right)^{v-1} \theta_{1}\left(\ln \frac{u}{L_{k}^{\star}}\right)^{1-v}
\end{aligned}
$$

By property (6) of Lemma 2.5, the equality $l_{k}^{\star}=l^{\star}\left(x_{k}, L_{k}\right)=r_{k}$ holds. Consequently, we get the estimates

$$
L_{k} \leq 2 \sigma u^{-v / \alpha} L_{k}^{\star v / \alpha} \leq 2 \sigma u^{-v / \alpha}\left(C^{\star}+1\right)^{v / \alpha} l_{k}^{\star v / \alpha}=b r_{k}^{\nu / \alpha}
$$

The images of $B\left(x_{k}, r_{k}\right)$ under the mapping $f$ cover the set $f\left(F_{i, j} \cap U\left(x_{0}, s\right)\right)$. From (4.18) and property (P2), we conclude that

$$
\begin{aligned}
\mathscr{H}_{\rho}^{\alpha}\left(f\left(F_{i, j} \cap U\left(x_{0}, s\right)\right)\right) & \leq \sum_{k} \operatorname{diam}_{\rho}^{\alpha}\left(f\left(B\left(x_{k}, r_{k}\right)\right)\right) \\
& \leq 2^{\alpha} \sum_{k} L_{k}^{\alpha} \leq 2^{\alpha} b^{\alpha} \sum_{k} r_{k}^{v}<2^{\alpha} b^{\alpha} \varepsilon .
\end{aligned}
$$

Thus $\mathcal{H}_{\rho}^{\alpha}\left(f\left(F_{i, j} \cap U\left(x_{0}, s\right)\right)\right)=0$, which contradicts (1.3). 
Some similar estimates of the index of a point were obtained in [21] for the case $\mathbb{G}=\mathbb{R}^{n}$.

COROLLARY 4.6. Let $f: \Omega \rightarrow \mathbb{G}$ be a nonconstant quasiregular mapping, and let $F \subset B_{f}$ be a continuum. Then

$$
\inf _{x \in F} i(x, f) \leq \frac{\theta_{1}}{\theta_{2}} v^{\nu-1} K_{I}(f)
$$

and set $E=\left\{x \in B_{f}: i(x, f) \geq v^{\nu-1}\right\}$ is totally disconnected.

Proof. If $F$ is a continuum, then $\mathscr{H}_{\rho}^{1}(F)>0$ and the first assertion follows from Theorem 4.5 with $\alpha=1$.

If we assume that $E$ contains a continuum, then we get the contradiction with the first statement.

Corollary 4.7. Let $\Omega \subset \mathbb{G}$ be an open connected set, and let $f: \Omega \rightarrow \mathbb{G}$ be a nonconstant quasiregular mapping such that $B_{f}$ is not empty. Then

$$
\inf _{x \in F} i(x, f)<K_{I}(f) \frac{\theta_{2}}{\theta_{1}}\left(\frac{v}{v-(m+1)}\right)^{v-1} .
$$

Here $m$ is the index of the last vector space of the graduated Lie algebra $\varphi$ of $\mathbb{C}$.

PROOF. Corollary 4.7 follows from Theorems 4.5 and 3.3.

AcKnowledgment. This work was supported by Projects Fondecyt, Chile, no. 1020067 and no. 1030373.

\section{REFERENCES}

[1] B. Bojarski and T. Iwaniec, Analytical foundations of the theory of quasiconformal mappings in $\mathbf{R}^{n}$, Ann. Acad. Sci. Fenn. Ser. A I Math. 8 (1983), no. 2, 257324.

[2] A. V. Černavskiū, Finite-to-one open mappings of manifolds, Mat. Sb. (N.S.) 65 (107) (1964), 357-369 (Russian).

[3] _ Addendum to the paper "Finite-to-one open mappings of manifolds", Mat. Sb. (N.S.) 66 (108) (1965), 471-472 (Russian).

[4] V. M. Chernikov and S. K. Vodop'yanov, Sobolev spaces and hypoelliptic equations. I, Siberian Adv. Math. 6 (1996), no. 3, 27-67.

[5] __ Sobolev spaces and hypoelliptic equations. II, Siberian Adv. Math. 6 (1996), no. 4, 64-96.

[6] W.-L. Chow, Über Systeme von linearen partiellen Differential gleichungen erster Ordnung, Math. Ann. 117 (1939), 98-105 (German).

[7] L. J. Corwin and F. P. Greenleaf, Representations of Nilpotent Lie Groups and Their Applications. Part I. Basic Theory and Examples, Cambridge Studies in Advanced Mathematics, vol. 18, Cambridge University Press, Cambridge, 1990.

[8] N. S. Dairbekov, Mappings with bounded distortion on Heisenberg groups, Sibirsk. Mat. Zh. 41 (2000), no. 3, 567-590, ii (Russian), translation in Siberian Math. J. 41 (2000), no. 3, 465-486. 
[9]

[12] J. Heinonen, Calculus on Carnot groups, Fall School in Analysis (Jyväskylä, 1994), Report, vol. 68, University of Jyväskylä, Jyväskylä, 1995, pp. 1-31.

[13] J. Heinonen and I. Holopainen, Quasiregular maps on Carnot groups, J. Geom. Anal. 7 (1997), no. 1, 109-148.

[14] J. Heinonen, T. Kilpeläinen, and O. Martio, Nonlinear Potential Theory of Degenerate Elliptic Equations, Oxford Mathematical Monographs, Clarendon Press, Oxford University Press, New York, 1993.

[15] J. Heinonen and P. Koskela, Definitions of quasiconformality, Invent. Math. 120 (1995), no. 1, 61-79.

[16] I. Holopainen and S. Rickman, Quasiregular mappings of the Heisenberg group, Math. Ann. 294 (1992), no. 4, 625-643.

[17] L. Hörmander, Hypoelliptic second order differential equations, Acta Math. 119 (1967), 147-171.

[18] A. Koranyi and H. M. Reimann, Foundations for the theory of quasiconformal mappings on the Heisenberg group, Adv. Math. 111 (1995), no. 1, 1-87.

[19] I. Markina, Extremal length for quasiregular mappings on Heisenberg groups, to appear in J. Math. Anal. Appl.

[20] - On coincidence of $p$-module of a family of curves and $p$-capacity on the Carnot group, Rev. Mat. Iberoamericana 19 (2003), no. 2, 1-18.

[21] O. Martio, A capacity inequality for quasiregular mappings, Ann. Acad. Sci. Fenn. Ser. A I Math. (1970), no. 474, 1-18.

[22] O. Martio, S. Rickman, and J. Väisälä, Definitions for quasiregular mappings, Ann. Acad. Sci. Fenn. Ser. A I Math. (1969), no. 448, 1-40.

[23] _ Topological and metric properties of quasiregular mappings, Ann. Acad. Sci. Fenn. Ser. A I Math. (1971), no. 488, 1-31.

[24] G. D. Mostow, Quasi-conformal mappings in $n$-space and the rigidity of hyperbolic space forms, Inst. Hautes Études Sci. Publ. Math. (1968), no. 34, 53-104.

[25] A. Nagel, E. M. Stein, and S. Wainger, Balls and metrics defined by vector fields. I. Basic properties, Acta Math. 155 (1985), no. 1-2, 103-147.

[26] P. Pansu, Croissance des boules et des géodésiques fermées dans les nilvariétés [Growth of balls and closed geodesics in nilmanifolds], Ergodic Theory Dynam. Systems 3 (1983), no. 3, 415-445 (French).

[28] E. A. Polecki1, The method of moduli for nonhomeomorphic quasiconformal mappings, Mat. Sb. (N.S.) 83 (125) (1970), no. 2 (10), 261-272 (Russian).

[29] Yu. G. Reshetnyak, Mappings with bounded distortion as extremals of integrals of Dirichlet type, Sibirsk. Mat. Ž. 9 (1968), no. 3, 652-666 (Russian).

[30] _ Spatial Mappings with Bounded Distortion, Izdatel'stvo "Nauka" Sibirskoe Otdelenie, Novosibirsk, 1982 (Russian). 
[31] Space Mappings with Bounded Distortion, Translations of Mathematical Monographs, vol. 73, American Mathematical Society, Rhode Island, 1989.

[32] S. Rickman, Quasiregular Mappings, Ergebnisse der Mathematik und ihrer Grenzgebiete (3), vol. 26, Springer-Verlag, Berlin, 1993.

[33] U. Srebro, Quasiregular mappings, Advances in Complex Function Theory (Proc. Sem., Univ. Maryland, College Park, Md, 1973-1974), Lecture Notes in Math., vol. 505, Springer-Verlag, Berlin, 1976, pp. 148-163.

[34] E. M. Stein, Some problems in harmonic analysis suggested by symmetric spaces and semi-simple groups, Actes du Congrès International des Mathématiciens (Nice, 1970), Tome 1, Gauthier-Villars, Paris, 1971, pp. 173-189.

[35] _ Harmonic Analysis: Real-Variable Methods, Orthogonality, and Oscillatory Integrals, Princeton Mathematical Series, vol. 43, Princeton University Press, New Jersey, 1993.

[36] R. S. Strichartz, Sub-Riemannian geometry, J. Differential Geom. 24 (1986), no. 2, 221-263.

[37] S. K. Vodop'yanov, Monotone functions and quasiconformal mappings on Carnot groups, Sibirsk. Mat. Zh. 37 (1996), no. 6, 1269-1295 (Russian), translation in Siberian Math. J. 37 (1996), no. 6, 1113-1136.

[38] Quasiconformal mappings on Carnot groups and their applications, Dokl. Akad. Nauk 347 (1996), no. 4, 439-442 (Russian).

[39] _ Mappings with bounded distortion and with finite distortion on Carnot groups, Sibirsk. Mat. Zh. 40 (1999), no. 4, 764-804 (Russian), translation in Siberian Math. J. 40 (1999), no. 4, 644-677.

[40] _, 9 -differentiability on Carnot groups in different topologies and related topics, Proceedings on Analysis and Geometry (Russian) (Novosibirsk Akademgorodok, 1999), Izdat. Ross. Akad. Nauk Sib. Otd. Inst. Mat., Novosibirsk, 2000, pp. 603-670 (Russian).

[41]__ Closedness of classes of mappings with bounded distortion on Carnot groups, Mat. Tr. 5 (2002), no. 2, 92-137 (Russian).

[42] S. K. Vodop'yanov and I. Markina, Local estimates of a variation of mappings with bounded s-distortion on the Carnot groups, The 12-th Siberian School on the Algebra, Geometry, Analysis and Mathematical Physics, Izdatel'stvo Instituta Matematiki, Novosibirsk, 1998, pp. 28-53 (Russian).

[43] S. K. Vodop'yanov and A. D. Ukhlov, Approximately differentiable transformations and the change of variables on nilpotent groups, Sibirsk. Mat. Zh. 37 (1996), no. 1, 70-89 (Russian), translation in Siberian Math. J. 37 (1996), no. $1,62-78$.

[44] Sobolev spaces and $(P, Q)$-quasiconformal mappings of Carnot groups, Sibirsk. Mat. Zh. 39 (1998), no. 4, 776-795 (Russian), translation in Siberian Math. J. 39 (1998), no. 4, 665-682.

Irina Markina: Departamento de Matemática, Universidad Técnica Federico Santa María, Casilla 110-V, Valparaíso, Chile

E-mail address: irina.markina@mat.utfsm.c1 


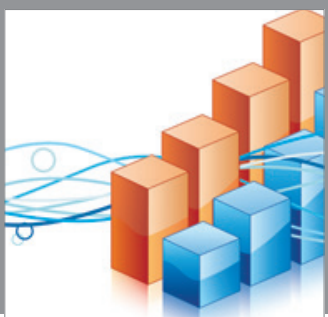

Advances in

Operations Research

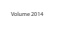

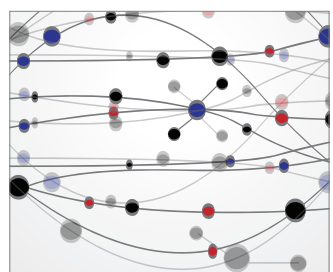

\section{The Scientific} World Journal
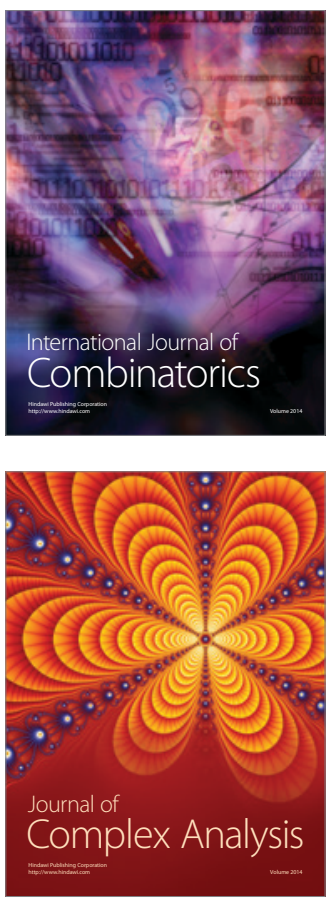

International Journal of

Mathematics and

Mathematical

Sciences
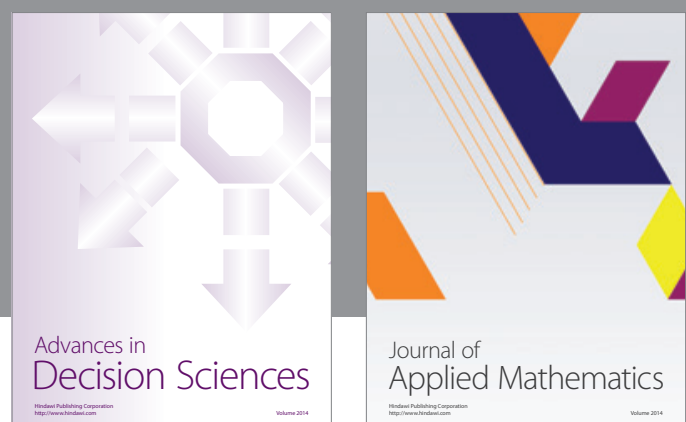

Journal of

Applied Mathematics
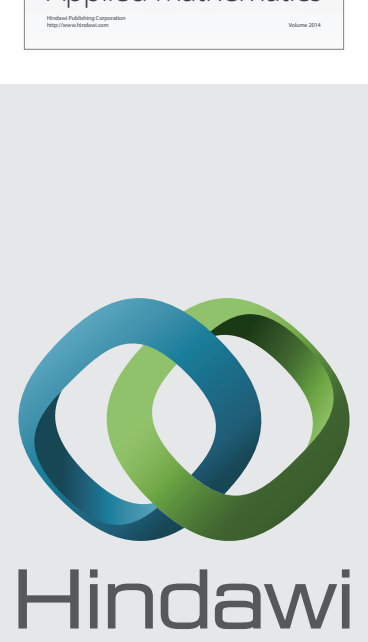

Submit your manuscripts at http://www.hindawi.com
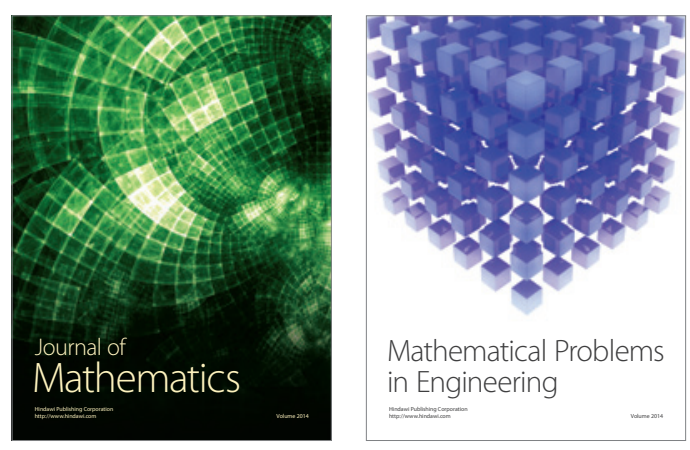

Mathematical Problems in Engineering
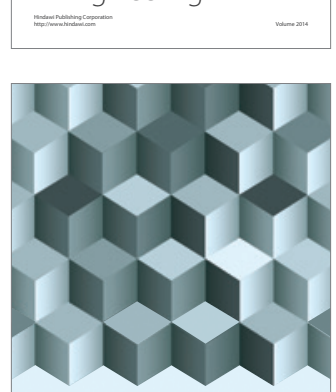

Journal of

Function Spaces
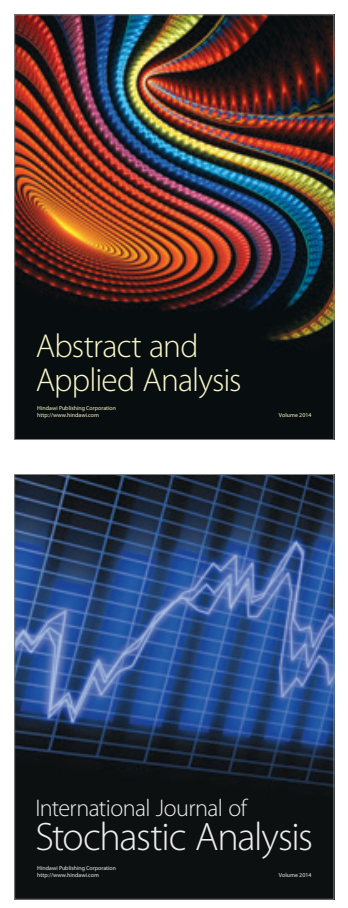

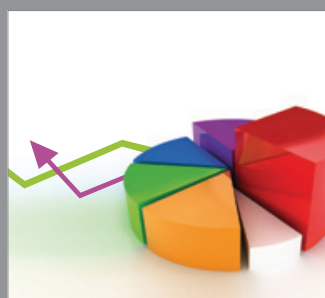

ournal of

Probability and Statistics

Promensencen
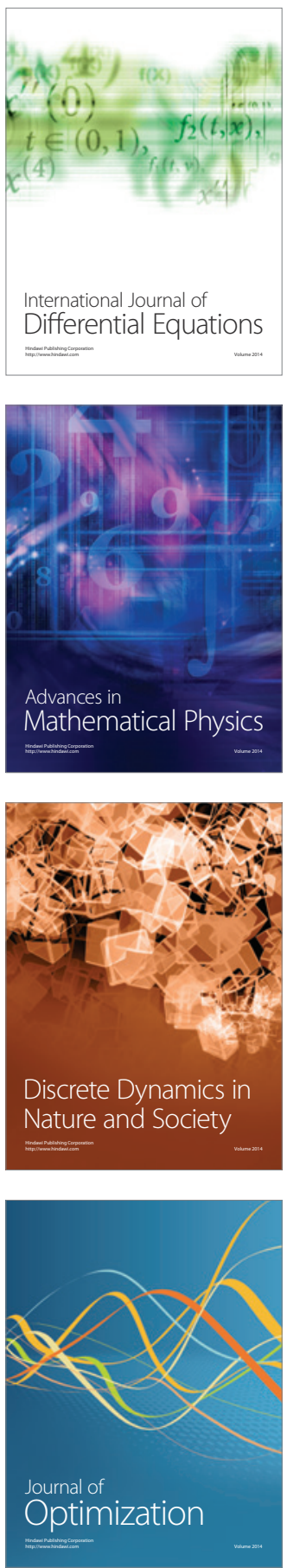